\title{
Reimplante tardío de un incisivo central superior avulsionado: reporte de caso
}

\author{
Late reimplant of avulsed upper central incisor: case report
}

\author{
Giovanny García Solano', Fabriciano José Martínez Heleno²
}

Para citar este artículo: García G, Martínez FJ. Reimplante tardío de un incisivo central superior avulsionado: Reporte de caso. UstaSalud, 2017;16: 89-96

Licencia Creative Commons

\section{(c) (1) () $\Theta$} lo tanto, los lectores pueden acceder libremente a los artículos en su formato .pdf, igualmente podrán descargarlos y difundirlos; sin embargo no podrán modificarlos o alterarlos, adicionalmente se debe reconocer la autoría de las personas que figuran en las publicaciones, pero estas no podrán comercializadas.

\begin{abstract}
1 Fundación Oftalmológica de Santander (FOSCAL), Servicios de Odontología, Bucaramanga, Colombia.

${ }^{2}$ Alianza Diagnostica S.A., Servicios de Odontología, Bucaramanga, Colombia.
\end{abstract}

Autor de correspondencia: Giovanny García Solano

Correo electrónico: gigasolandroid@gmail.com

\section{RESUMEN}

La avulsión dentaria es el resultado más severo y con peor pronóstico de los traumatismos dento-alveolares. El reimplante dentario constituye un desafío para el odontólogo, debido a la exigencia del manejo y el seguimiento estricto a los protocolos que pueden determinar un resultado exitoso a largo plazo. En este reporte de caso se presenta el seguimiento clínico y radiográfico durante 5 años, de un reimplante dental tardío de un incisivo central superior a las 7 horas después de su avulsión, en un adolescente de 16 años de edad, proveniente de Bucaramanga. La evolución del caso mostró un estado favorable a pesar de las condiciones limitadas de tiempo y conservación de la pieza dentaria.

Palabras clave: Avulsión de diente, reimplante dental, reporte de caso.

\section{ABSTRACT}

The most severe dento-alveolar trauma is dental avulsion, its forecast is questionable in many cases. Dental reimplantation is a challenge for the dentist, due to the strict management and strict fulfilment of protocols that can determine a successful long-term outcome. This case report shows a 5-years clinical and radiographic follow-up of an avulsed right maxillary central incisor reimplanted 7 hours later in a 16 -year-old teenager from Bucaramanga. The evolution of the case showed a positive status in spite of the limited conditions of time and conservation of the tooth.

Keywords: Tooth avulsion, tooth reimplantation, case report. 


\section{INTRODUCCIÓN}

Las lesiones dentales traumáticas tienen una alta frecuencia, tanto en la dentición permanente como en la decidua, siendo esta ultima la más afectada ${ }^{1,2}$; dichas lesiones podrían clasificarse si se tiene en cuenta el tipo de estructura anatómica que se ve comprometida, la clasificación que establece la Organización Mundial de la Salud (OMS) ${ }^{3}$ contempla lesiones de tejidos duros dentales y pulpares, lesiones de los tejidos periodontales, lesiones de los maxilares y lesiones de encía y mucosas. En la avulsión dental varias estructuras como la pulpa, el ligamento periodontal y el hueso alveolar se pueden afectar, debido a que la pieza dental se desaloja por completo de su alveolo; es necesario reconocer que la ausencia de una pieza anterior implica inconvenientes para el corte de algunos alimentos, igualmente representa una limitación para la fonación e impacta negativamente la calidad de vida en relación con la salud bucal y la percepción estética ${ }^{4}$.

La prevalencia de estos eventos varía de acuerdo con varios factores y puede tener relación con la edad del individuo y sus actividades o comportamientos, un estudio realizado en Iran por Rouhani et al..$^{5}$ en 778 participantes con una edad promedio de $10,3 \pm 2,1$ determinó que el $22,9 \%$ presentó signos de algún tipo de trauma dentoalveolar y de esos 178 casos el 16,9\% correspondía a episodios de avulsión. En la ciudad de Medellín se encontró que de 549 sujetos evaluados, 11 registraban antecedentes de avulsión en su historia clínica ${ }^{6}$, resultados similares se encontraron en el Hospital de la Misericordia de Bogotá, donde 15 de 668 pacientes que consultaron por servicios de salud bucal, acudieron por avulsión ${ }^{7}$.

La presentación de estos casos en la consulta odontológica si bien no es igual de frecuente a otras afecciones bucales, sí requiere de atención y observación especial, puesto que el paquete neurovascular de la pulpa se rompe a nivel del foramen apical y el tejido puede necrosarse fácilmente ${ }^{8}$, por esto es indispensable que los pacientes que sufren este tipo de trauma consulten en el menor tiempo posible con un odontólogo para la evaluación y resolución del caso; la inadecuada intervención o la aplicación errónea de los protocolos podría perjudicar el pronóstico del diente en caso de que se desee reimplantar, por lo tanto, el profesional debe contar con competencias y habilida- des basadas en protocolos científicos que orienten un tratamiento oportuno y conveniente con el fin de lograr un buen resultado a largo plazo.

Existen algunos factores y algunas situaciones que aumentan el riesgo de fracaso, Donaldson y Kinironsen el $2001^{9}$ concluyeron con los resultados de su estudio que la contaminación, el daño coronal y la conservación del diente son tres factores determinantes en el inicio temprano de la reabsorción en dientes reimplantados; otro desenlace común que puede ocurrir después de la reimplantación es la anquilosis que resulta de la pérdida de células presentes en el ligamento periodontal ${ }^{10}$. El propósito de la terapéutica es mitigar los efectos al ligamento periodontal y a la pulpa, es difícil evitar las afecciones celulares al ligamento periodontal y se pueden agravar fácilmente de acuerdo con el tiempo y las condiciones en las que se encuentre el diente en medio extraoral (deshidratación), debido a que en medio seco el fibroblasto no tiene gran longevidad; entonces resulta muy importante instaurar medidas de seguridad que aminoren la reabsorción ${ }^{11}$.

Por otro lado, la investigación sugiere que la calcitonina tiene un efecto favorable en la detección de la reabsorción externa una vez se reimplanta una pieza dental. Los biofosfonatos y el floruro de estaño al parecer tienen efectos similares en cuanto a la reabsorción radicular ${ }^{12,13}$.

El objetivo de este reporte de caso es exponer el seguimiento durante cinco años de un incisivo central superior derecho reimplantado a las 7 horas después de su avulsión.

\section{PRESENTACIÓN DEL CASO}

\section{Reporte del odontólogo general}

Paciente de género masculino de 16 años de edad, sin enfermedades sistémicas, ni antecedentes alérgicos o quirúrgicos; asistió acompañado por su acudiente a la consulta odontológica después de haber estado en observación por 6 horas en el servicio hospitalario de urgencias, donde recibió atención primaria, terapia analgésica (se desconoce el fármaco) y terapia inmunológica con toxoide tetánico (de acuerdo con los protocolos de atención primaria de urgencias del 
lugar donde fue atendido). Según el testimonio del acudiente el paciente sufrió un accidente casero, que incluyó un trauma directo en la región anterior de su rostro, durante la anamnesis se descartaron situaciones de violencia intrafamiliar. En la exploración física extraoral se evidenciaron múltiples lesiones traumáticas con apósitos de atención hospitalaria y el labio superior presentaba un evidente edema; al examen intraoral, se encontró relación molar y canina clase I bilateral y un arco tipo 2, sobremordida vertical y horizontal normales (considerando el incisivo central superior izquierdo), con apiñamiento anterosuperior e inferior moderado; además se evidenciaron defectos del esmalte de tipo opacidades difusas compatibles con fluorosis dental. En el momento de la consulta también se observaron subluxaciones de los dientes continuos y fracturas no complicadas que comprometían únicamente esmalte. El diente 11 presentó avulsión y no se apreciaron fracturas en tablas óseas alveolares en ninguna de las arcadas, sin embargo la encía se observaba ligeramente edematizada.

Infortunadamente, en la valoración primaria de los servicios de urgencias hospitalarias no siempre se encuentra un odontólogo presente en el momento de la atención del paciente con trauma dentoalveolar, perdiéndose así gran cantidad de información y detalles del accidente; no obstante, durante la anamnesis se informó que el diente había sido conservado en medio seco en una servilleta de papel, lo cual podría a ver reducido el éxito del reimplante de manera significativa; sin embargo, se decidió reimplantar el diente siguiendo las recomendaciones de la Asociación Americana de Endodoncistas para el tratamiento de lesiones causadas por trauma dentoalveolar ${ }^{14}$, que establece un protocolo específico para aquellos dientes avulsionados que han permanecido más de sesenta segundos en un medio extraoral seco. Al considerar el pronóstico del caso por el tiempo transcurrido después de la avulsión, las condiciones de almacenamiento y los requerimientos tanto del acudiente como del paciente se procedió a realizar el reimplante, informando previamente la necesidad de un tratamiento de endodoncia a las dos semanas posteriores, e informando verbalmente y en el consentimiento informado un posible fracaso a corto/largo plazo.

$\mathrm{Al}$ momento de evaluar el diente avulsionado no se observó fractura en sus tercios radiculares, debi- do a que no se conservó en un medio húmedo este se encontraba totalmente reseco, se apreció una fractura coronal no complicada del ángulo disto-incisal; no se tomó radiografía del área de la lesión por el estado de hiperalgesia del paciente quien se encontraba consternado y ansioso por el procedimiento a realizarse. Se hizo el bloqueo infiltrativo con Lidocaína al $2 \%$ con epinefrina, se examinó el alveolo para descartar fragmentos óseos y fracturas en sus paredes, se eliminaron los desechos presentes y el ligamento periodontal necrótico, se realizó el lavado y la irrigación del alveolo con solución salina para eliminar el coágulo contaminado, el diente fue lavado con solución salina para eliminar residuos y fragmentos de tejido blando; posteriormente fue sumergido en solución de fluoruro de sodio acidulado durante 10 minutos, nuevamente el diente fue lavado con solución salina y, posteriormente, se comprobó el ajuste del diente dentro del alveolo y su alineación clínica. Para la estabilidad se utilizó una férula semirrígida con nylon, abarcando desde el diente 13 al diente 23, se instauró terapia antibiótica por 7 días con Doxiciclina de 100mg, al paciente se le indicó que durante las siguientes dos semanas debía someterse a una dieta blanda, usar un cepillo de dientes con cerdas suaves después de cada comida y utilizar un enjuague bucal con clorhexidina $(0,12 \%)$ dos veces al día durante una semana; finalmente el adolescente fue remitido al especialista en Endodoncia para continuar el tratamiento de conducto.

\section{Reporte del endodoncista}

Paciente remitido a la consulta especializada de Endodoncia por antecedentes de trauma dentoalveolar con cuadro clínico de 7 días de evolución por avulsión del incisivo central superior derecho y por reimplante tardío; al examen clínico se observó el diente 11 con férula semirrígida con Nylon en consecuencia por el antecedente de avulsión y la reimplantación tardía, se decidió iniciar tratamiento endodóntico. Con la aprobación por escrito del acudiente se hizo endodoncia uniradicular del diente 11; después de realizar la apertura cameral, fue permeabilizado el conducto, se preparó el conducto radicular con instrumentación rotatoria reciprocante Wave One $e^{\circledast}$ lima L, y técnica Crown Down, la longitud de trabajo fue de 21 $\mathrm{mm}$ desde el borde incisal (Figura 1a), el conducto fue irrigado con hipoclorito de Sodio al 5,25\%, EDTA al $17 \%$, Clorhexidina al $0,2 \%$ y se obturó con cemento de Grossman (GROSSFAR ${ }^{\oplus}$ Root Cannal Sealer) y conos 
de gutapercha (Gutta-Percha Points, Dentsply Maillefer) obturados con condensación vertical con técnica de onda continua (Figura 1b).

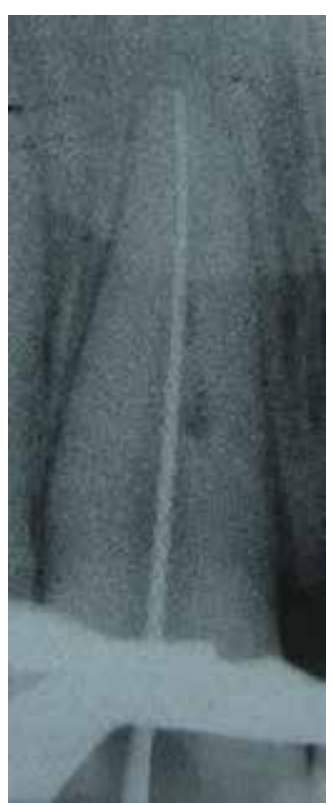

$1 \mathrm{a}$

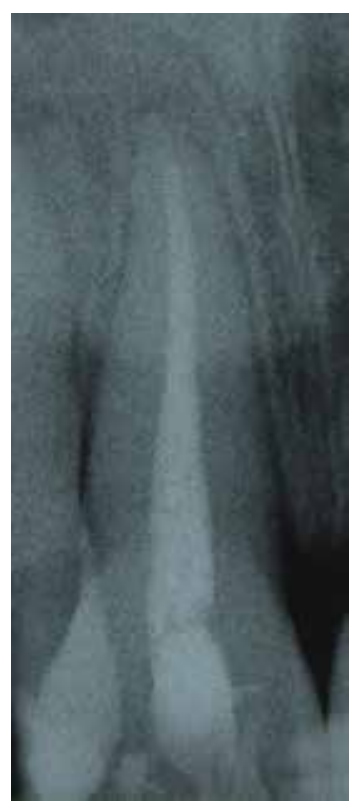

$1 b$

Figuras 1a y $1 b$. Procedimiento de endodoncia del diente 11, la figura la muestra la radiografía de conometría mientras que la $1 \mathrm{~b}$ la radiografía de penachos.

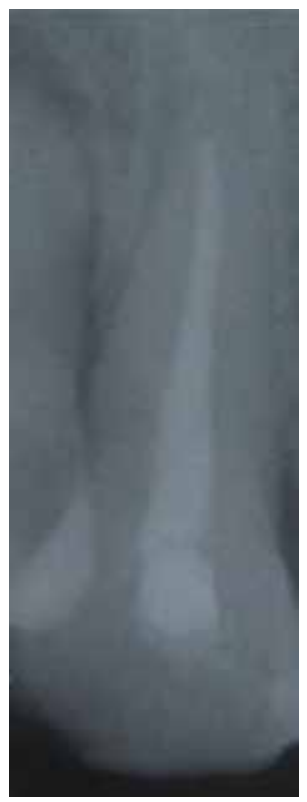

$2 \mathrm{a}$

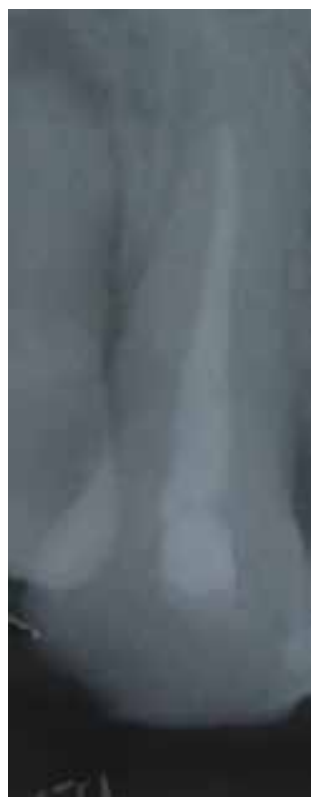

$2 \mathrm{~b}$

\section{Control radiográfico por 5 años}

El paciente asistió a controles anuales y recibió inspección clínica y radiográfica, durante el análisis radiográfico no se apreciaron áreas radiolucidas perirradiculares indicativas de reabsorción radicular progresiva inflamatoria externa, o pérdida de la lámina dura indicativa de anquilosis, no se apreciaron signos de enfermedad periodontal y el diente no presentaba movilidad. A los cinco años después del reimplante se evidencian resultados positivos ante las pocas expectativas del caso particularmente por las condiciones desfavorables del tiempo transcurrido desde el momento de la avulsión y el medio de conservación. Durante los años 2013 y 2014 (Figuras 2a y 2b) se tomaron radiografías periapicales convencionales con equipo estándar; en los años 2016 y 2017 se utilizó radiovisografo InstaRay $1.5^{\circledR}$ (Figuras $2 \mathrm{c}$ y $2 \mathrm{~d}$ ). La imagen radiográfica en el diente 11 al control después de 2 años, se visualiza pérdida de la continuidad tercio incisal coronal compatible con fractura no complicada, también se presenta un leve ensanchamiento del espacio del ligamento periodontal posiblemente debido al tratamiento de ortodoncia que está recibiendo el paciente (Figura 2d). No se aprecian signos de reabsorción inflamatoria en sus tercios apicales, además la cresta ósea y el hueso alveolar se encuentran normales sin lesiones radiolucidas, igual que en el periapice; se

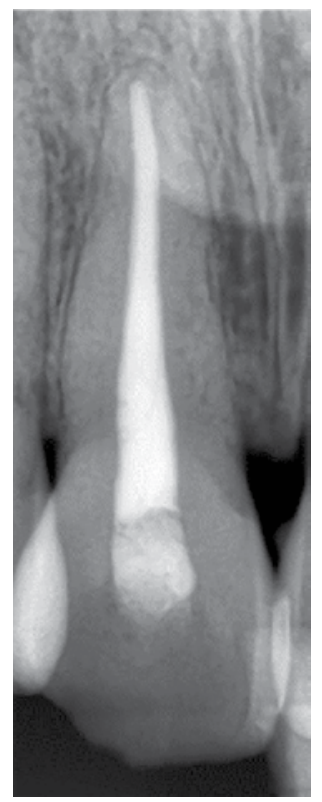

$2 c$

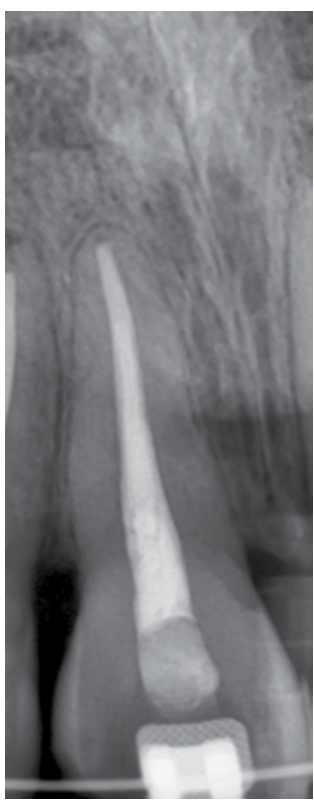

$2 \mathrm{~d}$

Figura 2a 2b 2c y 2d. Control radiográfico al primer año después del reimplante en el 2013 (2a), después de dos años en el 2014 (2b), después de cuatro años en el 2016 (2c) y a los cinco años en el 2017 (2d). 
descartan fisuras o fracturas en sus tercios radiculares, sin embargo, el diente 12 presenta ensanchamiento del ligamento periodontal y una leve lesión radiolucida en su ápice (Figura 2d).

En cuanto al estado de la obturación radicular del diente 11 esta se encontró homogénea, compacta y hermética, pero se pueden observar ligeros cambios radiográficos en el diente como signos leves de reabsorción inflamatoria en el tercio medio radicular del lado mesial, sin embargo, el tamaño es de pocos milímetros y también es evidente un desvanecimiento del espacio ligamento periodontal en el lado mesial en tercio cervical radicular; adicional a estos cambios no se encontraron otros hallazgos radiográficos de mayor importancia que pudiesen comprometer el pronóstico del diente. En el examen realizado en 2016 se hace un registro clínico del caso (Figura 3 ) donde se observa el margen gingival del diente 11 ligeramente desplazado hacia apical, adicional a esto los tejidos periodontales circundantes se encuentran bajo los parámetros de normalidad.

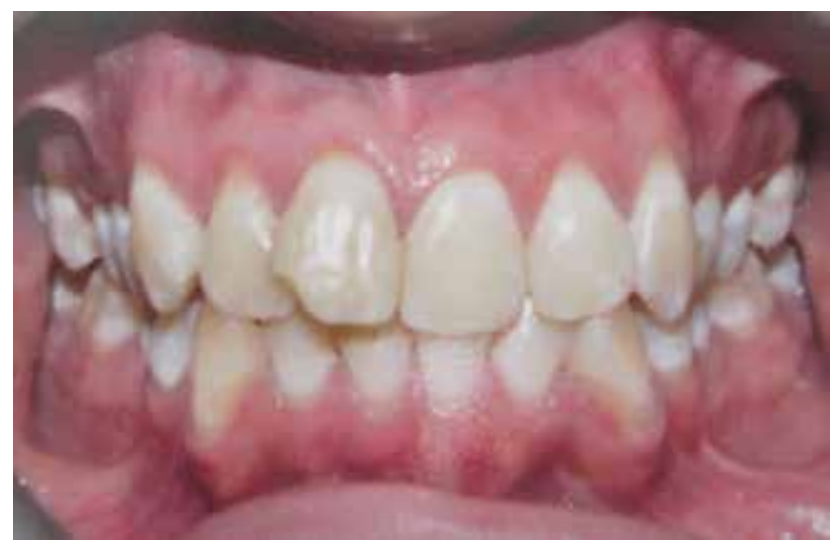

Figura 3. Seguimiento clínico a los cuatro años después del reimplante.

En la última radiografía de control que corresponde al examen realizado en 2017, el paciente presentaba aditamentos de un tratamiento de ortodoncia recientemente iniciado (Figura 2d); el paciente será citado con la misma regularidad para conocer el estado del diente durante y al finalizar dicho tratamiento ortodóntico. El primer autor de este reporte de caso informó detalladamente al especialista en ortodoncia acerca de los procedimientos realizados con respecto al diente 11 con el fin de que el especialista proporcionara un tratamiento apropiado y cuidadoso.

\section{DISCUSIÓN}

El presente reporte mostró el caso de un incisivo central superior derecho reimplantado después de casi 7 horas de ser avulsionado, a los cinco años de ejecutar el procedimiento el estado del diente parece ser favorable y su función satisface las mismas necesidades que los dientes adyacentes según el reporte del paciente y la evaluación clínica. La obturación radicular mostró condiciones adecuadas aun en el quinto año y se descartaron signos de lesión periapical al realizar el análisis radiográfico. En este caso particular el tratamiento de conductos se vuelve indispensable debido a la formación completa del ápice, el extenso periodo transcurrido hasta la reimplantación de la pieza dentaria y las condiciones asépticas de almacenamiento; según Heithersay ${ }^{15}$ cuando el diente avulsionado ha terminado su formación radicular ya existe una baja probabilidad de reestablecer elementos pulpares viables y el resultado a largo plazo en la mayoría de los casos suele ser la necrosis pulpar; en adición Bastos et al. ${ }^{16}$ concluyeron en su estudio publicado en 2014 que evaluó 205 dientes reimplantados, que el tiempo transcurrido entre la reimplantación y el inicio de la terapia endodóntica afectó progresivamente la gravedad y la prevalencia de reabsorciones inflamatorias externas, teoría que se relaciona con los resultados de Werder, von Arx, Chappuis ${ }^{8}$ en 2011, puesto que el tratamiento de conductos fue ejecutado a las dos semanas en todos los dientes analizados en su investigación y ninguno de ellos desarrolló reabsorción.

En las ultimas radiografías presentadas en caso abordado se logra apreciar mejor la leve reabsorción radicular externa (figuras $2 \mathrm{c} \mathrm{y} 2 \mathrm{~d}$ ) la cual puede guardar una relación con el almacenamiento en medio seco según varias investigaciones $s^{8,17,18}$, en especial la de Rhouma, McMahon y Welbury ${ }^{17}$ en Escocia donde de 16 dientes que se mantuvieron en un medio seco solo 6 de ellos pudieron continuar con su función en boca y no fracasaron después del reimplante, resultado que se soporta con un OR de 4,13 (IC 95\% 1,33 - 12,80). No obstante, en el presente reporte la reabsorción observada no comprometió la permanencia del diente en la arcada superior, desenlace que podría deberse a la inmersión del diente en fluoruro de sodio, tal y como lo reportan varias investigaciones en animales ${ }^{19-21}$. 
A pesar del pronóstico desfavorable que se conoce para aquellos dientes que se mantienen por un periodo extenso fuera de algún medio húmedo ${ }^{8,9,22,23}$ se ha intentado encontrar resultados exitosos a largo plazo como en los reportes de caso de Cobankara y Ungor ${ }^{24}$ y de Cho y Cheng ${ }^{25}$ donde los dientes permanentes fueron en ambos casos conservados en un medio no fisiológico por 7 días y 18 horas, respectivamente, en ningún documento se demostró un éxito definitivo a largo plazo y en conclusión sugieren que estos reimplantes tardíos de dientes que han permanecido en ambientes secos se realicen con los propósitos de contrarrestar la pérdida de hueso alveolar y ofrecer una solución transitoria mientras se logra concretar un tratamiento protésico definitivo; en el presente caso se conocían claramente las desventajas del tiempo de intervención y del almacenamiento del diente, sin embargo, en el último análisis radiográfico las condiciones del diente fueron aceptables, evidentemente no se puede descartar el fracaso a largo plazo, por esto se seguirá realizando la observación pertinente del diente en cuestión.

Acerca de la utilización de compuestos de proteínas derivadas de matriz del esmalte (EMD) o de algún factor de crecimiento fibroblástico básico (bFGF, FGF-2) aún se siguen realizando estudios en animales a pesar de los efectos positivos que se han demostrado hasta la fecha ${ }^{26-28}$; pero los resultados indican que pueden evitar en algunos casos la reabsorción radicular y favorecen la regeneración de las células vitales del ligamento periodontal, sin embargo en la investigación realizada por Tuna et al. ${ }^{27}$ en el 2015 con 18 dientes de perros beagle se evidencia que el uso de bFGF favoreció a la formación de nuevo ligamento periodontal, previno la aparición de anquilosis y de reabsorción en reimplantaciones tardías mientras que los dientes sumergidos en EMD mostraron reabsorción y otros efectos indeseados.

Kahler y Heithersay ${ }^{29}$ en el 2008 en su trabajo de revisión encontraron que respecto al tipo de férula y el tiempo apropiado en los casos de avulsión la mayoría de autores sugieren emplear una férula funcional, que permita cierto grado de movilidad con el fin de reducir el riesgo de anquilosis y organizaciones como la Academia Americana de Odontología Pediátrica y la Asociación Americana de Endodoncistas recomiendan mantener la férula por un periodo mínimo de siete días y máximo de catorce ${ }^{30}$, a diferencia de la IADT (International Association for Dental Trauma) que aconseja un tiempo de dos semanas. En el presente estudio la férula fue retirada a los 14 días, periodo que se sitúa dentro de los tiempos recomendados; durante el seguimiento radiográfico se observaron algunos signos leves de anquilosis muy habituales en estos casos según lo reportado en la literatura.

Para concluir, se puede decir que la avulsión dental traumática es una urgencia en el consultorio odontológico y es habitual principalmente en poblaciones jóvenes. Para conseguir una adecuada reinserción y fijación de la pieza en el alvéolo es necesario conservar la vitalidad del ligamento periodontal. En estos casos, aspectos como el tiempo, la cuidadosa manipulación y preservación del diente avulsionado en medios adecuados son esenciales para el éxito del tratamiento a largo plazo. Cuando se presente un caso de avulsión dental similar al expuesto en esta publicación, debe considerarse que a mayor tiempo fuera de un medio fisiológico, peor pronóstico a largo plazo tendrá la pieza dentaria, y si el clínico decidiese realizar un reimplante de un diente que lleva varias horas en medio seco deberá reconocer que la ubicación del diente en el alveolo podría ser una solución únicamente provisional hasta que pueda instaurarse una solución definitiva.

\section{AGRADECIMIENTOS}

El equipo investigador quiere expresar su agradecimiento a los doctores Jaime Omar Moreno Monsalve y Emelina Toloza, docentes de la Universidad Santo Tomás Seccional Bucaramanga, por sus recomendaciones para el artículo. De igual forma a la doctora Ángela Patricia Ortiz García, ortodoncista por su interés y seguimiento en el caso clínico y, finalmente al doctor José Fernando Pieruccini Arias por la oportunidad de publicar el artículo en la Revista Ustasalud.

\section{REFERENCIAS}

1. Zaleckiene V, Peciuliene V, Brukiene V, Drukteinis S. Traumatic dental injuries: etiology, prevalence and possible outcomes. Stomatologija. 2014;16(1):7-14.

2. Andreasen JO, Lauridsen E, Gerds TA, Ahrensburg SS. Dental Trauma Guide: A source of evidence-based treat- 
ment guidelines for dental trauma. Dental Traumatology. 2012;28(5):345-50. doi: 10.1111/j.1600-9657.2011.01059.

3. World Health Organization. Application of the International Classification of Diseases to Neurology: ICD-NA Second Edition: World health organization; 1997.

4. AL-Omiri MK, Karasneh JA, Lynch E, Lamey P, Clifford TJ. Impacts of missing upper anterior teeth on daily living. Int Dent J. 2009;59(3):127-32. doi: 10.1922/ IDJ_1994ALOmiri06.

5. Rouhani A, Movahhed T, Ghoddusi J, Mohiti Y, Banihashemi E, Akbari M. Anterior traumatic dental injuries in East Iranian school children: prevalence and risk factors. Iran Endod J. 2015;10(1):35-8.

6. Amaya EMP, Correa LR, Jiménez LDP, Garzón NS, Arcila SO, Arcila O. Análisis de los incidentes de trauma dentoalveolar atendidos en el servicio de urgencias de una institución docente asistencial de Medellín (Colombia) 2007-2012. Revista Nacional de Odontología. 2014;10(18):21-8. doi: 10.16925/od.v10i18.718.

7. Duarte Albarracín LM. Prevalencia y caracterización del trauma dentoalveolar en niños y adolescentes atendidos por el servicio de salud oral en la Fundación HOMI Hospital de la Misericordia en el período febrero 2010 - enero 2013 [Trabajo de grado] Bogotá: Universidad Nacional de Colombia; 2013.

8. Werder P, von Arx T, Chappuis V. Treatment outcome of 42 replanted permanent incisors with a median follow-up of 2.8 years. Schweiz Monatsschr Zahnmed. 2011;121(4):312-20.

9. Donaldson M, Kinirons M. Factors affecting the time of onset of resorption in avulsed and replanted incisor teeth in children. Dental Traumatology. 2001;17(5):201-5. doi: 10.1034/j.1600-9657.2001.170503.x.

10. Campbell KM, Casas MJ, Kenny DJ. Ankylosis of traumatized permanent incisors: pathogenesis and current approaches to diagnosis and management. J Can Dent Assoc. 2005;71(10):763-8.

11. Andreasen JO, Andreasen F, Skeie A, Hjørting-Hansen E, Schwartz O. Effect of treatment delay upon pulp and periodontal healing of traumatic dental injuries-a review article. Dental Traumatology. 2002;18(3):116-28. doi: 10.1034/j.1600-9657.2002.00079.x.

12. Donado Manotas JE, Solano G, Armando J, Osorio Ordóñez M, Guerrero F. Efectividad de la calcitonina para detener la reabsorción externa posterior a un reimplante. Rev Fed Odontol Colomb. 1993;45(179):19-31.
13. Levin L, Bryson E, Caplan D, Trope M. Effect of topical alendronate on root resorption of dried replanted dog teeth. Dental Traumatology. 2001;17(3):120-6. doi: 10.1034/j.1600-9657.2001.017003120.x.

14. American Association of Endodontists. The Recommended Guidelines of the American Association of Endodontists for the Treatment of Traumatic Dental Injuries. 2004:2-4.

15. Heithersay GS. Replantation of avulsed teeth. A review. Aust Dent J. 1975;20(2):63-72.

16. Bastos JV, de Souza Côrtes, Ilma, Goulart EMA, Colosimo EA, Gomez RS, Dutra WO. Age and timing of pulp extirpation as major factors associated with inflammatory root resorption in replanted permanent teeth. J Endod. 2014;40(3):366-71. doi: 10.1016/j.joen.2013.10.009.

17. Rhouma O, McMahon A, Welbury R. Early prognostic indicators and outcome prediction model for replanted avulsed teeth. European Archives of Paediatric Dentistry. 2012;13(4):203-9. doi: 10.1007/BF03262871.

18. Petrovic B, Marković D, Peric T, Blagojevic D. Factors related to treatment and outcomes of avulsed teeth. Dental Traumatology. 2010;26(1):52-9. doi: 10.1111/j.1600-9657.2009.00836.x.

19. Shulman L, Gedalia I, Feingold R. Fluoride concentration in root surfaces and alveolar bone of fluoride-immersed monkey incisors three weeks after replantation. J Dent Res. 1973;52(6):1314-16. doi: $10.1177 / 00220345730520062701$

20. Bjorvatn K, Weiss M. Effect of topical application of fluoride, cortisone and tetracycline on reimplanted rat molars. Fasett. 1971;1:27-31.

21. Bjorvatn K, Massler M. Effect of fluorides on root resorption in replanted rat molars. Acta Odontol Scand. 1971;29(1):17-29.

22. Hammarström L, Blomlöf L, Lindskog S. Dynamics of dentoalveolar ankylosis and associated root resorption. Dental Traumatology. 1989;5(4):163-75. doi: 10.1111/ j.1600-9657.1989.tb00354.x.

23. Pohl Y, Tekin U, Boll M, Filippi A, Kirschner H. Investigations on a cell culture medium for storage and transportation of avulsed teeth. Australian Endodontic Journal. 1999;25(2):70-5.

24. Cobankara FK, Ungor M. Replantation after extended dry storage of avulsed permanent incisors: report of a case. Dental Traumatology. 2007;23(4):251-6. doi:10.1111/j.1600-9657.2005.00425.x. 
25. Cho S, Cheng AC. Replantation of an avulsed incisor after prolonged dry storage: a case report. Journal-Canadian Dental Association. 2002;68(5):297-300.

26. Barbizam JV, Massarwa R, Silva LAB, Silva RAB, NelsonFilho P, Consolaro A, et al. Histopathological evaluation of the effects of variable extraoral dry times and enamel matrix proteins (enamel matrix derivatives) application on replanted dogs' teeth. Dental Traumatology. 2015;31(1):29-34. doi: 10.1111/edt.12131.

27. Tuna EB, Arai K, Tekkesin MS, Seymen F, Gencay K, Kuboyama $\mathrm{N}$ et al. Effect of fibroblast growth factor and enamel matrix derivative treatment on root resorption after delayed replantation. Dental Traumatology. 2015;31(1):49-56. doi: 10.1111/edt.12141.
28. Araujo M, Hayacibara R, Sonohara M, Cardaropoli G, Lindhe J. Effect of enamel matrix proteins (Emdogain ${ }^{\circ}$ ) on healing after re-implantation of "periodontally compromised" roots. J Clin Periodontol. 2003;30(10):855-61. doi: 10.1034/j.1600-051X.2003.00400.x.

29. 29. Kahler B, Heithersay GS. An evidence-based appraisal of splinting luxated, avulsed and root-fractured teeth. Dental Traumatology. 2008;24(1):2-10. doi: $10.1111 /$ j.1600-9657.2006.00480.x.

30. Jordán F, Sossa H, Estrada JH. Protocolo de manejo de diente permanente avulsionado para el servicio de salud oral de la Fundación Hospital La Misericordia y la Facultad de Odontología de la Universidad Nacional de Colombia, sede Bogotá (2011). Univ Odontol. 2012;31(66):185-210. 